\title{
Mapping evidence on the distribution of human papillomavirus-related cancers in sub-Saharan Africa: scoping review protocol
}

\author{
Bridget K. M. Lekoane, Tivani P. Mashamba-Thompson and Themba G. Ginindza*
}

\begin{abstract}
Background: Despite the introduction of HPV vaccines, the incidence of HPV-related cancers (cervical, penile, anal, vulvar, vagina, head, and neck) in sub-Saharan Africa has been rising. The increasing incidence of these HPV-related cancers has been attributed to changes in lifestyle-related risk factors, most notably sexual behavior. The main objective of this study is to map evidence on the distribution of HIV-related cancers in sub-Saharan Africa (SSA).

Methods and analysis: We will conduct a scoping review to explore, describe, and map literature on the distribution of HPV-related cancers in sub-Saharan Africa. The primary search will include peer-reviewed and review articles. The list of references from included studies will also be searched. The search will be performed using EBSCOhost platform by searching the following databases within the platform: Academic search complete, health source: nursing/academic edition, CINAHL with full text, PubMed, Science Direct, Google scholar and World Health Organization (WHO) library databases, and gray literature. The researcher will search the articles using keywords, from the included studies; abstract and full articles will be screened by two independent reviewers. The screening will be guided by the inclusion and exclusion criteria. A thematic content analysis will be used to present the narrative account of the reviews, using NVivo version 10.
\end{abstract}

Discussion: We anticipate finding relevant literature on the distribution of HPV-related cancers in sub-Saharan Africa. The study findings will help reveal research gaps to guide future research.

Systematic review registration: PROSPERO CRD42017062403.

Keywords: HPV-related cancers, Risk factors, Prevalence, Incidence, Mortality, Trends, HIV

\section{Background}

The International Agency for Research on Cancer (IARC) defined human papilloma virus (HPV)-associated cancers as a specific cellular type of cancers diagnosed in a part of the body where HPV DNA is found [1]. HPV-related cancers are found on the parts of the body such as cervix, vulva, vagina, anal, penile, and oropharynx [2]. Per worldwide estimates, HPV infection is responsible for $5.2 \%$ of all cancers [3, 4]. Cancers associated with HPV are more prevalent in developing countries as compared to developed countries [5]. HPV is implicated in $99.7 \%$ of cervical cancer, $85 \%$ of anal cancers, $20 \%$ of oropharyngeal cancers, and $50 \%$ of cancers of the vulva, vagina, and penis

\footnotetext{
* Correspondence: Ginindza@ukzn.ac.za

Discipline of Public Health Medicine, School of Nursing and Public Health, University of KwaZulu-Natal, Mazisi Kunene Road, Durban 4041, South Africa
}

cases worldwide [6]. According to estimates, cervical cancer is the fourth most common cancer among women with 572,624 new cases worldwide, which around $85 \%$ occurred in less developed regions. Around 266,000 females died of cervical cancer, accounting for $7.5 \%$ of all female cancer deaths [7].

WHO developed guidelines for prevention and control of cervical cancer; the main elements in the guideline were to vaccinate 9-13-year-old girls with two doses of HPV vaccine, use HPV tests to screen women for cervical cancer prevention, and communicate more widely to reach a wider audience e (http://www.who.int/ reproductivehealth/RHUpdate/en/index.html). HPV vaccines are now broadly used in the prevention of cervical cancer; quadrivalent and bivalent are the two HPV vaccines developed towards mitigating the burden of 
cervical cancer. They have been pre-qualified by the World Health Organization (WHO) and approved by national government in many counties [8]. Also, the adoption of Papanicolaou(Pap) smear screening programs became successful and resulted in declining cervical cancer rates in Canada and the USA, but the incidence of other HPV-related cancers has been increasing $[9,10]$. These increasing incidences of HPVrelated cancers have been attributed to changes in lifestyle-related risk factors, most notably sexual behavior [10].

A scoping review of the literature regarding the distribution of HPV-related cancers in sub-Saharan Africa (SSA) is to be conducted. Interventions aimed at preventing and controlling cervical cancer in high endemic regions such as SSA are encouraged. However, research studies aimed at helping improve the efficiency of implementing these interventions are required. The aim of the study is to map evidence on the distribution of HPV-related cancers in subSaharan Africa. It is anticipated that the results of this study will reveal research gaps to guide future primary research and scoping reviews on HPV-related cancers in sub-Saharan Africa.

\section{Methods and materials}

The current scoping review protocol is to be performed according to Preferred Reporting Items for Systematic Reviews and Meta-Analysis Protocols (PRISMA-P) guidelines [11] (Additional file 1: Figure S1), and the protocol is registered with PROSPERO under registration number CRD42017062403 and accessible via this link: https:// www.crd.york.ac.uk/PROSPERO/display_record.asp?ID= CRD42017062403.

\section{Scoping review framework}

The framework proposed review by Arksey $\mathrm{H}$ and O'Malley will be influential in conducting the scoping review [12]. The framework involves (I) identifying the research question, (II) identifying relevant studies, (III) study selection, (IV) charting the data, and (V) collating, summarizing, and reporting the results.

\section{Identifying the research question}

The major research question is "What is the evidence on the burden of HPV-related cancers in Sub-Saharan Africa?"

Research sub-questions are as follows:

- What is the burden of HPV-related cancers in subSaharan Africa with estimation on the prevalence, incidence, and mortality?
- What are the risk factors associated with HPVrelated cancers?

- What are the trends of HPV-related cancers?

- What is the association of HIV and HPV-related cancers?

Eligibility criteria Studies will be selected according to the PEO (population, exposure, and outcomes) framework outlined below (Table 1).

\section{Identifying relevant studies}

There will be no date and language restrictions applied on literature search. We will perform a keyword search from the following electronic databases: PubMed; World Health Organization (WHO) library; Science Direct; Google scholar; EBSCOhost platform for the following databases: Academic search complete, Health Source: Nursing/Academic Edition, and CINAHL with full text. We will use the following keywords while searching the above databases: HPV-related cancers, risk factors, prevalence, incidence, trends, HIV, and sub-Saharan Africa. Boolean terms AND and OR will be used to separate the keywords during the search. Mesh terms (Medical Subject Headings) will also be included in the search. The researcher will do a hand search of eligible studies from the list of references of included studies.

\section{Study selection and eligibility}

Following title screening from the above databases, articles with relevant title to the subject of the research will be uploaded on the Endnote X7 X7.7.1 software. Search results from different electronic databases will be combined in a single EndNote library (Appendix 1). Studies which do not address the research question and the duplicates of the same records will be excluded. From the included studies, abstract and full articles will be screened by two

Table 1 PEO framework for eligibility of research question

\begin{tabular}{ll}
\hline Criteria & Determinants \\
\hline $\begin{array}{l}\text { Population and their } \\
\text { problem }\end{array}$ & $\begin{array}{l}\text { Individuals with HPV-related cancers in Sub-Sa- } \\
\text { haran Africa } \\
\text { Exposure }\end{array}$ \\
Outcome & $\begin{array}{l}\text { 1. Mortality } \\
\text { 2. Incidence } \\
\text { 3. Prevalence } \\
\text { 4. Risk factors(cervical cancer, anal cancer, penile } \\
\text { cancer, vulva cancer, and head and neck cancers) } \\
\text { 5. Interplay with HIV } \\
\text { 6. Trends }\end{array}$ \\
\end{tabular}


independent reviewers. An abstract screening form with questions will be developed based on the review eligibility criteria. The screening will be guided by the eligibility criteria. Discrepancies between reviewers at the abstract stage will be resolved through consensus and will involve a third reviewer. Discrepancies between reviewers at full article stage will be resolved by involving a third screener.

The relevant studies will be identified with guidance from the inclusion and exclusion criteria which were formulated according to the research questions.

\section{Eligibility criteria Inclusion criteria}

- Study will include individuals with HPV-related cancers

- All study designs with relevant intervention

- Studies that focus on HPV-related cancers; prevalence, incidence, mortality, risk factors, and trends

- Studies that focus on HIV and HPV-related cancers.

\section{Exclusion criteria}

- Studies that do not focus on HPV-related cancers

- Studies that do not focus on humans

- Qualitative studies will also be excluded

PRISMA flow chart will be used to report the screening results.

Table 2 will be used to show the results of the titles searched from different databases.

PRISMA flow chart (Additional file 1: Figure S1) will be used to summarize the inclusion and exclusion criteria.

\section{Charting the data}

Data from included studies will be extracted using an extraction form (Appendix 2). A data charting form will be developed and will be used to determine which variables to extract that will help to answer the research question. The extraction form will continually be updated. The form will include the following: author with date, study title, study design, study setting, population, study aim, intervention,

Table 2 Database search record

\begin{tabular}{lllll}
\hline $\begin{array}{l}\text { Date of } \\
\text { search }\end{array}$ & $\begin{array}{l}\text { Search } \\
\text { engine }\end{array}$ & $\begin{array}{l}\text { Keyword } \\
\text { searched }\end{array}$ & $\begin{array}{l}\text { Number of } \\
\text { articles found }\end{array}$ & $\begin{array}{l}\text { Number articles } \\
\text { eligible }\end{array}$ \\
\hline
\end{tabular}

percentages, outcomes of the study, key findings, and comments (Table 3).

\section{Collating, summarizing, and reporting the results.}

The aim of this study is to map the existing evidence and to summarize the findings as presented in articles. A narrative account of findings from existing literature will be presented through thematic content analysis. Literature will be extracted and structured around the following outcomes: prevalence, incidence, mortality, risk factors, trends, and HIV. The resulting themes will be analyzed and results will inspect the relationship between the findings and the research question. The meaning of the findings will be considered as they relate to the overall study purpose and the implications of these findings for future research, policy, and practice.

\section{Quality assessment}

The quality of the evidence obtained from the studies will be assessed to make sure the study design is appropriate for the research objectives and the studies are reported well and to eliminate risk of bias. A quality appraisal tool which focuses on the study methods, the Mixed Method Quality Appraisal Tool (MMAT) Version 2011, will be used [13]. The tool will be used to examine the quality of an article looking at the following aspects: the appropriateness of the aim of the study, adequacy and methodology, study design, participant recruitment, data collection, data analysis, presentation of findings, authors' discussions, and conclusions.

\section{Discussion}

The current scoping review aims to map available evidence regarding the distribution of HPV-related cancers, the risk factors, and its association with HIV and AIDS in sub-Saharan Africa in order to reveal research

Table 3 Data charting form

Author and date
Study design
Study setting
Population
- Average age
- Sample size
Aims
Intervention
Outcome
Key findings
Conclusion
Comment


gaps in this area. High-risk HPV types are the cause of all cervical cancers, anogenital cancers including the vulva, and anal and penile cancers $[14,15]$, as well as head and neck cancers [16]. Sub-Saharan Africa has the highest incidence of HPV and cervical cancer in the world [17]. HPV-related cancers are rising and they are a major public health concern exacerbating current disease burden in sub-Saharan Africa [18]. The burden of HPV-associated diseases is substantially increased where there is a HIV-1 co-infection [19], and with diverse HIV/AIDS epidemic in sub-Saharan Africa, the burden of HPV-related cancers might still rise. The Sustainable Development Goals (SDG) include targets relevant to women's cancers, including a one-third reduction in premature mortality from noncommunicable diseases through prevention and treatment by 2030 and achievement of universal health coverage, with access to quality essential health-care services and access to safe, effective, quality, and affordable essential medicines and vaccines for all [20].

The focus of this review is on HPV-related cancers, hence excluding studies that do no focus on HPVrelated cancers and humans as the data will be irrelevant and will not report according to the set research questions. The study will focus on all individuals with HPVrelated cancers regardless of gender. There is a latency period of 10 years or more between the initial high-risk HPV infection and the development of cancer; hence, there are no date restrictions on the literature search. Since the study is aimed on mapping the distribution of the HPV-related cancer, qualitative studies will be excluded in this review.

Despite information about HPV vaccine and testing within cervical cancer screening programs, it has been shown that patients' and public's knowledge of HPVrelated cancers is still very little [21, 22]. Therefore, the results of this study will help address this knowledge gap. It is anticipated that our study findings will help to strengthen a need for policy implementation for prevention of HPV-related cancers and raise more awareness of HPV-related cancers. Also, government and stakeholders can be able to ensure that HPV vaccines are introduced everywhere as part of a coordinated and comprehensive strategy to prevent cervical cancer and other diseases caused by HPV. There will also be proper implementation of education on reducing behaviors that increase the risk of acquiring HPV infection, training of health workers and information to women about screening, diagnosis, and treatment of precancerous lesions and cancer especially in underdeveloped and financially constrained countries in sub-Saharan Africa. The finding will also help strengthen the need of HPV vaccines to be included in national immunization programs in the whole of sub-Saharan Africa.

\section{Appendix 1}

Table 4 How results from databases will be recorded

\begin{tabular}{llll}
\hline Date of search & $\begin{array}{l}\text { Search } \\
\text { engine used }\end{array}$ & Keyword search & $\begin{array}{l}\text { Number of } \\
\text { publications } \\
\text { retrieved }\end{array}$ \\
\hline $18 / 07 / 2017$ & PubMed & ("epidemiology"[Subheading] & 1268 \\
& OR "epidemiology"[All Fields] & \\
& OR "prevalence"[All Fields] OR & \\
& "prevalence"[MeSH Terms]) & \\
& AND hpv[All Fields] AND \\
& related[All Fields] AND \\
& ("neoplasms"[MeSH Terms] & \\
& OR "neoplasms"[All Fields] & \\
& OR "cancers"[All Fields])) & \\
& AND ("loattrfull text"[sb] & \\
& AND "humans"[MeSH & \\
& Terms]) \\
& &
\end{tabular}

\section{Appendix2}

Table 5 Sample data extraction form

Study details
Author/year
Objectives
Participants (characteristics/total number)
Setting/context
Interventions
Search details
Sources searched
Years of included studies
Number of studies included/types of studies included
Country of origin of included studies
Appraisal
Appraisal instruments used
Appraisal rating
Analysis
Method of analysis
Outcome assessed
Results/findings
Significance/direction

\section{Additional file}

Additional file 1: Figure S1. PRISMA flow diagram. (DOCX 33 kb)

\section{Abbreviations}

AIDS: Acquired immunodeficiency syndrome; DNA: Deoxyribonucleic acid; HIV: Human immunodeficiency virus; HPV: Human papilloma virus; IARC: International Agency for Research on Cancer; MMAT: Mixed Methods Appraisal Tool; PRISMA-P: Preferred Reporting Items for Systematic Reviews and Meta-Analysis Protocols; SDG: Sustainable Development Goals;

SSA: Sub-Saharan Africa; WHO: World Health Organization

\section{Acknowledgements}

The authors would like to thank the University of KwaZulu-Natal (UKZN) for the provision of resources towards this review and the UKZN Systematic Review Unit for the training and technical support.

Funding

Not applicable 


\section{Availability of data and materials}

All data generated or analyzed during this study will be included in the published systematic review article.

\section{Authors' contributions}

BL conceptualized the study under the supervision of TG and TPM-T and designed data collection methods. BL, TG, and TPM-T contributed to writing the first draft of the manuscript. All authors critically reviewed and approved of the final manuscript.

\section{Ethics approval and consent to participate}

Not applicable

\section{Consent for publication}

Not applicable

\section{Competing interests}

The authors declare that they have no competing interests.

\section{Publisher's Note}

Springer Nature remains neutral with regard to jurisdictional claims in published maps and institutional affiliations.

Received: 18 May 2017 Accepted: 7 November 2017

Published online: 17 November 2017

\section{References}

1. International Agency for Research on Cancer. IARC monographs on the evaluation of carcinogenic risks to humans. Volume 90: Human papillomaviruses. Lyon, France: International Agency for Research on Cancer, World Health Organization; 2007.

2. Shiels MS, Kreimer AR, Coghill AE, Darragh TM, Devesa SS. Anal cancer incidence in the United States, 1977-2011: distinct patterns by histology and behavior. Cancer Epidemiol Biomarkers Prev. 2015;24:1548-56.

3. Parkin DM. The global health burden of infection-associated cancers in the year 2002. Int J Cancer. 2006;118:3030-44.

4. Parkin DM, Bray F. Chapter 2: the burden of HPV-related cancers. Vaccine. 2006:24(Suppl 3):S3/11-25.

5. Agosti JM, Goldie SJ. Introducing HPV vaccine in developing countries—-key challenges and issues. N Engl J Med. 2007;356:1908-10. doi:10.1056/NEJMp078053 PMID:17494923.

6. Walboomers JM, Jacobs MV, Manos MM, Bosch FX, Kummer JA, Shah KV et al. Human papilloma virus is a necessary cause of invasive cancer worldwide. J Pathol. 1999;189:12-9. doi:10.1002/(SICI)1096-9896(199909)189: 1<12: AID-PATH431>3.0.CO:2-F PMID:10451482.

7. Ferlay, J., Soerjomataram, I., Ervik, M., Dikshit, R., Eser, S., Mathers, C., Rebelo, M., Parkin, D.M., Forman, D., Bray, F.: GLOBOCAN 2012 v1.0, Cancer incidence and mortality worldwide: IARC CancerBase No. 11 [online]. International Agency for Research on Cancer, Lyon (France) 2013. Available from WWW: http://globocan.iarc.fr.

8. Schiffman M, Wacholder S. Success of HPV vaccination is now a matter of coverage. Lancet Oncol. 2012;13:10-2. doi:10.1016/S1470-2045(11)70324-2 PMID:22075169.

9. Hatcher J, ed. Cervical cancer: 2010 report on cancer statistics in Alberta Edmonton (AB): Cancer Care, Alberta Health Services; 2012. Available: www. albertahealthservices.ca/poph/hi-poph-surv-cancer-cervical-2010.pdf (accessed accessed 2013 Jun. 20)

10. Chaturvedi AK. Beyond cervical cancer: burden of other HPV-related cancers among men and women. J Adolesc Health. 2010;46(Suppl):S20-6.

11. Moher D, Shamseer L, Clarke M, Ghersi D, Liberati A, Petticrew M, Shekelle P, Stewart LA. Preferred reporting items for systematic review and metaanalysis protocols (PRISMA-P) 2015 statement. Syst Rev. 2015:4(1):1.

12. Arksey H, O'Malley L. Scoping studies: towards a methodological framework. Int J Soc Res Methodology. 2005;8(1):19-32.

13. Pluye P, Robert E, Cargo M, Bartlett G, O'Cathain A, Griffiths F, Boardman F, Gagnon M, Rousseau M. Proposal: A mixed methods appraisal tool for systematic mixed studies reviews in.2011.http://mixedmethodsappraisa Itoolpublic.pbworks.com/w/page/24607821/ FrontPage. Accessed 12 Jan 2016.

14. Crum CP, et al. Pathobiology of vulvar squamous neoplasia. Curr Opin Obstet Gynecol. 1997;9:63-9.

15. Kayes $\mathrm{O}$, et al. Molecular and genetic pathways in penile cancer. Lancet Oncol. 2007:8:420-9.
16. Mannarini L, Kratochvil V, Calabrese L, et al. Human papillomavirus (HPV) in head and neck region: review of literature. ActaOtorhinolaryngol Ital. 2009:29:119-26.

17. De Vuyst H, Alemany L, Lacey C, Chibwesha CJ, Sahasrabuddhe V, Banura C, Denny L, Parham GP. The burden of human papillomavirus infections and related diseases in sub-Saharan Africa. Vaccine. 2013;31(Suppl. S5):F32-46.

18. Baxi SS, Shuman AG, Corner GW, et al. Sharing a diagnosis of HPV-related head and neck cancer: the emotions, the confusion, and what patients want to know. Head Neck. 2013:35:1534-41.

19. Pantanowitz $L$, Michelow P. Review of human immunodeficiency virus (HIV) and squamous lesions of the uterine cervix. Diagn Cytopathol. 201 1;39:65-72.

20. UN. Sustainable Development Goals. 2015. https://sustainabledevelopment. un.org/sdg3 (Accessed Sept 30, 2016).

21. Hendry M, Pasterfield D, Lewis R, et al. Are women ready for the new cervical screening protocol in England? A systematic review and qualitative synthesis of views about human papillomavirus testing. Br J Cancer. 2012;107:243-54.

22. Hendry M, Lewis R, Clements A, et al. "HPV? Never heard of it!": a systematic review of girls' and parents' information needs, views and preferences about human papillomavirus vaccination. Vaccine. 2013;31:5152-67.

\section{Submit your next manuscript to BioMed Central and we will help you at every step:}

- We accept pre-submission inquiries

- Our selector tool helps you to find the most relevant journal

- We provide round the clock customer support

- Convenient online submission

- Thorough peer review

- Inclusion in PubMed and all major indexing services

- Maximum visibility for your research

Submit your manuscript at www.biomedcentral.com/submit
Ciomed Central 